\title{
Étude microclimatique de l'effet de la sécheresse sur l'évaporation d'une plantation de pins maritimes et du sous-bois
}

\author{
P Berbigier ${ }^{*}$, A Diawara 1, D Loustau 2 \\ 1 INRA-Bordeaux, laboratoire de bioclimatologie, BP 81, 33883 Villenave d'Ornon cedex; \\ 2 INRA, laboratoire de sylviculture et d'écologie, Pierroton, 33610 Cestas, France
}

(Reçu le 30 mars 1990; accepté le 29 novembre 1990

\begin{abstract}
Résumé - L'évapotranspiration d'une plantation de pin maritime a été suivie dans les Landes pendant la sècheresse de l'année 1989 (mai-septembre), par une méthode microclimatique de bilan d'énergie-fluctuations et la méthode du débit de sève. Les méthodes mathématiques de description de la régulation stomatique au niveau des arbres et du sous-bois sont discutées. Les évolutions des moyennes horaires des conductances de surface et leurs relations avec le rapport de l'évapotranspiration au rayonnement net absorbé par chaque strate $\left(L E / R_{n}\right)$ pendant la phase diurne sont étudiées. Les valeurs journalières de $L E / R_{n}$ pour les pins semblent dépendre du stock d'eau du sol (0$75 \mathrm{~cm}$ ). Cependant, après le dessèchement initial, l'évapotranspiration des pins augmente même après des pluies qui affectent peu ce stock. Le rapport $L E / R_{n}$ du sous-bois reste élevé pendant toute l'expérience, sans doute par suite de la demande climatique relativement faible à ce niveau.
\end{abstract}

pin maritime / bilan d'énergie / fluctuation / évapotranspiration / résistance stomatique

Summary - A microclimatic study of the effect of drought on evapotranspiration in a Maritime pine stand and its understorey. The evapotranspiration of a Maritime pine stand was studied in the Landes forest (south-west France) during the severe drought of 1989 (May-September) by means of a microclimatic approach as well as sap flow measurements: heat flux was measured at 2 levels above the canopy and understorey by an eddy correlation method (2 monodimensional sonic anemometers coupled with fast-response temperature sensors), then latent heat flux was deduced from the energy balance of the 2 layers. Vertical profiles of air temperature and vapour pressure, leaf temperature measurements in the understorey, as well as assumptions regarding surface temperature of pine needles allow the computation of mean surface conductances of the tree canopy and understorey. In our experimental conditions, these conductances can be described fairly well by a linear function of the ratio of the net radiation absorbed by the layer on the air saturation deficit at its level $\left(R_{n} / D\right)$. This leads to a simple relationship between surface conductance and the ratio $L E / R_{n}$ (LE is the latent heat flux of each layer). The variations of daily LE/Rn values for pines seem to be related to the amount of water stored in the ground $(0-75 \mathrm{~cm})$. However, when drought is established, evapotranspiration increases after rainfalls, even if they do not obviously affect overall water storage. For the understorey, $\mathrm{LE} / \mathrm{R}_{\mathrm{n}}$ remains at high levels throughout the experiment, possibly due to the low climatic demand, as understorey net radiation is rather low.

maritime pine / energy balance / fluctuation / evapotranspiration / stomatal resistance

* Correspondance et tirés à part 


\section{INTRODUCTION}

Comme la plupart des végétaux, les conifères réagissent à un déficit d'alimentation en eau par une fermeture stomatique partielle : l'augmentation de la résistance du houppier aux transferts de vapeur d'eau, ramenée à des conditions microclimatiques comparables, constitue une des principales réponses physiologiques à la sécheresse. II en est de même pour la végétation du sous-bois.

La mesure de ces résistances, dont le terme principal est la résistance stomatique moyenne des feuilles ou aiguilles, peut se faire à l'aide de poromètres : cependant, cette technique bouleverse complètement l'environnement des stomates (section de la feuille, modification du microclimat), et, même dans un court laps de temps, il n'est pas exclu que les stomates réagissent à ces modifications. De plus, l'échantillonnage des feuilles et aiguilles est délicat. C'est pourquoi nous préférons estimer, à partir des méthodes micrométéorologiques, la résistance des différentes strates du couvert du flux de chaleur latente : nous avons fait l'hypothèse que celles-ci pouvaient se ramener à 2 surfaces sans épaisseur, l'une constituée par la surface totale des aiguilles, l'autre par celle des feuilles du sous-bois, placées à 2 niveaux moyens, et que les évaporations de ces 2 surfaces s'additionnaient pour donner l'évaporation totale du couvert.

Dans cette approche, la mesure fondamentale est celle de l'évapotranspiration des 2 niveaux. Pour les pins, nous avons la possibilité de comparer 2 méthodes, la mesure du flux de sève (Granier, 1985, 1987 a et b; Diawara et al, 1990) dont les possibilités et les limites sont assez bien connues, et une méthode micrométéorologique que nous décrivons plus en détail dans le texte. Au niveau du sous-bois, la même méthode micrométéorologique est appliquée; par ailleurs, au cours de la période expérimentale, quelques journées de mesure à l'aide d'une cage psychrométrique (El Hadj Moussa, 1989) sont effectuées, mais, les dates ne correspondant pas, nous n'en faisons pas plus ample mention.

Ce travail a été réalisé de mai à septembre 1989, période de sécheresse intense. Au vu des résultats présents, il nous est encore difficile de séparer, sur la réduction de l'évapotranspiration, les effets de la diminution de la réserve en eau du sol de ceux dus au vieillissement des feuilles et des aiguilles; aussi nous bornons-nous à faire une description qualitative des effets autres que micrométéorologiques, et à émettre quelques hypothèses. Toutefois, le but que nous cherchons à atteindre est une modélisation suffisamment précise de l'évolution des résistances du couvert pour obtenir un modèle prévisionnel de l'évapotranspiration de la forêt en fonction des paramètres du microclimat, modèle qui serait ensuite inclus dans un modèle hydrologique plus global.

\section{MATÉRIEL ET MÉTHODES}

\section{Site}

L'expérience s'est déroulée de mai à septembre 1989 sur une parcelle de pins maritimes (Pinus pinaster Ait) âgés de 20 ans, d'une hauteur moyenne de $13,6 \mathrm{~m}$, située à $20 \mathrm{~km}$ de Bordeaux, dans les Landes de Gironde. La parcelle est un carré de 16 ha limité par des chemins forestiers de $18 \mathrm{~m}$ de large et entourée par des parcelles similaires. Les vents dominants viennent d'ouest : dans cette direction, la forêt s'étend sur au moins $1 \mathrm{~km}$.

Les pins sont plantés sur des rangs de direction approximative NNE-SSW, espacés d'environ $4 \mathrm{~m}$; sur un rang, la distance entre 2 arbres est d'environ $3 \mathrm{~m}$. Leur indice foliaire est de 3,0 , 
ce qui correspond à une surface foliaire développée de $6 \mathrm{~m}^{2}$ par $\mathrm{m}^{2}$ de sol.

Le sous-bois est principalement constitué d'une graminée, la molinie (Molinia coerulea M) atteignant une hauteur moyenne de $0,7 \mathrm{~m}$, et qui est demeurée turgescente jusqu'à la fin des mesures, bien que l'épaisseur de feuilles sèches augmente progressivement. Son indice foliaire est de 1,2.

Le sol de la parcelle est un podzol sableux caractérisé par un lessivage intense et la formation d'un horizon d'accumulation humoferrugineux induré, à $0,65 \mathrm{~m}$ de profondeur moyenne. La profondeur maximale d'enracinement des pins est de $0,70 \mathrm{~m}$, celle de la molinie de $0,60 \mathrm{~m}$.

\section{Flux de sève}

La méthode du flux de sève appliquée aux arbres a été décrite par Granier (1985, 1987a, b). Son principe repose sur l'existence d'une relation entre l'écart de température entre 2 sondes insérées dans le xylème, l'une étant chauffée par effet Joule, et la vitesse de circulation de la sève dans celui-ci. Ses modalités particulières relatives à la parcelle expérimentale présente sont décrites par Diawara et al (1991). Le flux de sève est intégré sur des intervalles de $1 \mathrm{~h}$, de 0 à $24 \mathrm{~h}$, tout au long de la période expérimentale, à l'exception du j 215 qui est incomplet.

Résistance stomatique approchée $(\mathrm{s} / \mathrm{m})$

a

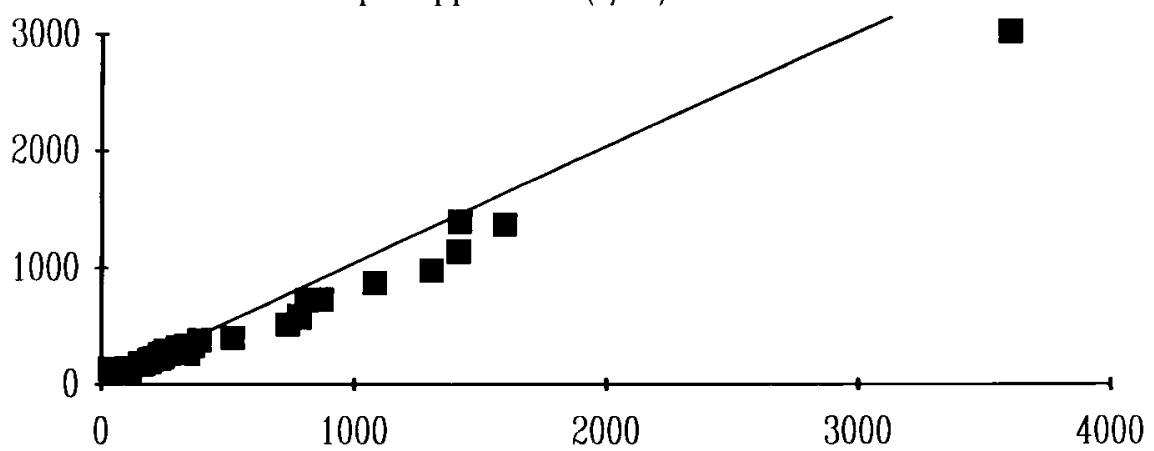

Résistance stomatique approchée $(\mathrm{s} / \mathrm{m})$

b

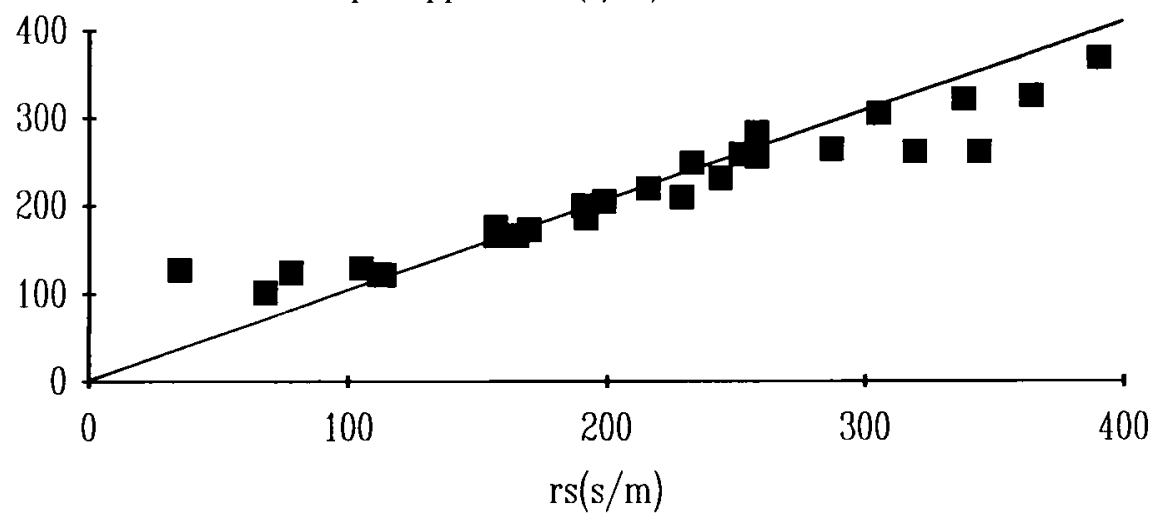

Fig 1. Comparaison entre la résistance stomatique approchée du couvert de molinie (équation 8) et le calcul complet de la résistance stomatique moyenne de ce couvert (équation 7 ). a ensemble des données; $b$ résistance stomatique comprise entre 0 et $400 \mathrm{~s} \cdot \mathrm{m}^{-1}$. En traits pleins : droite $Y=X$. 


\section{Méthode micrométéorologique}

Elle combine la mesure du flux de chaleur sensible $H$ à partir de la covariance des fluctuations de la composante verticale du vent et de la température de l'air, avec le bilan d'énergie, qui permet d'obtenir le flux de chaleur latente, à 2 hauteurs (au-dessus du couvert et au-dessus du sous-bois). Cette méthode est représentée mathématiquement par les équations (1), (2) et (3) :

$$
\begin{gathered}
H=\rho C_{p} \overline{W^{\prime} T H} \\
R_{n}=H+L E+S \\
S=G+J_{h}+J_{v}
\end{gathered}
$$

$A_{n}$ : densité de rayonnement net $\left(W . \mathrm{m}^{-2}\right)$

$H=$ densité de flux de chaleur sensible (W. $\left.\mathrm{m}^{-2}\right)$

$L E$ : densité de flux de chaleur latente (W. $\mathrm{m}^{-2}$ )

$S$ : stockage de chaleur dans le couvert et le sol (W. $\mathrm{m}^{-2}$ )

$G$ : stockage de chaleur dans le sol (W. $\left.\mathrm{m}^{-2}\right)$

$J_{h}$ : stockage de chaleur sensible dans l'air du couvert (W. $\left.\mathrm{m}^{-2}\right)$

$J_{v}$ : stockage de chaleur dans la biomasse (W. $\mathrm{m}^{-2}$ )

$\rho$ : masse volumique de l'air $\left(\mathrm{kg} \cdot \mathrm{m}^{-3}\right)$

$C_{p}$ : chaleur massique de l'air $\left(\mathrm{J} . \mathrm{kg}^{-1} \cdot{ }^{\circ} \mathrm{C}^{-1}\right)$

$w^{\prime}$ : fluctuation de la composante verticale de la vitesse du vent $\left(\mathbf{m} . \mathbf{s}^{-1}\right)$

$T$ : fluctuation de la température de l'air $\left({ }^{\circ} \mathrm{C}\right)$.

La présence d'une barre au-dessus d'une expression représente la valeur moyenne de celleci.

Contrairement à McCaughey et Saxton (1988) et Stewart (1988), nous ne faisons pas intervenir le stockage de vapeur d'eau dans le couvert dans le bilan d'énergie. En effet, l'augmentation de la teneur en vapeur d'eau de l'air ne correspond pas à un échange d'énergie s'il n'y a pas de changement de phase liquidevapeur; or, le flux d'énergie provoqué par le changement de phase initial (évapotranspiration des surfaces végétales) est déjà pris en compte par le terme $L E$ de l'équation (2).

Le calcul des termes de stockage $G, J_{h}$ et $J_{v}$ nécessite de décomposer le sol, l'air et la végétation en plusieurs parties (plusieurs strates horizontales pour chaque composante de la bio- masse et du sol) homogènes quant à leur capacité calorifique (produit de la densité par la chaleur spécifique) et à leur température. Le stockage de chaleur dans chacune de ces parties est le produit de sa capacité calorifique par la vitesse d'accroissement de sa température $\delta T / \delta t$. Ces valeurs partielles sont ensuite sommées pour obtenir les 3 termes de stockage.

Le stockage de chaleur dans le sol est apprécié à partir de profils de température (5 tubes verticaux portant latéralement des soudures de thermocouples à $1,4,9,16,25,36$ et $49 \mathrm{~cm}$ de profondeur, les soudures de référence étant groupées à $1 \mathrm{~m}$ ), de la composition (obtenue par analyse densitométrique) et de la teneur en eau du sol (Diawara, 1990). De même, le stockage de chaleur sensible dans l'air du couvert est estimé à partir de profils de température sèche (11 thermocouples ventilés de 0 à $18 \mathrm{~m}$ de hauteur). Le stockage dans les aiguilles et les feuilles est calculé à partir d'estimations de la biomasse verte et de mesures de sa teneur en eau, en faisant l'hypothèse que feuilles et aiguilles ont la même température que l'air ambiant à la même hauteur. Enfin, pour le stockage dans le tronc et les branches, nous avons équipé un arbre témoin de 8 capteurs de température, 3 à $2 \mathrm{~m}$ de hauteur, 3 à $6 \mathrm{~m}$, sous l'écorce, dans l'aubier et dans le cœur, et 2 dans une grosse branche, sous l'écorce et au centre. À partir d'une modélisation très simple et de la géométrie de l'arbre, de la connaissance des caractéristiques physiques du bois et de sa teneur en eau, la chaleur stockée peut être estimée. Tous ces calculs sont décrits en détail dans Amadou (1989), Diawara (1990) et Diawara et al (1991).

La stockage dans l'air et la végétation au niveau du sous-bois est pratiquement négligeable. Faute de données, l'effet de la litière n'est pas pris en compte.

Le rayonnement net au-dessus du couvert est mesuré par un bilanmètre placé à $18 \mathrm{~m}$ du sol; au-dessus du sous-bois, il est estimé à partir de la moyenne des réponses de 6 bilanmètres placés à $1,75 \mathrm{~m}$ du sol, dans le rectangle limité par 4 arbres. Diawara et al (1991) montrent qu'une moyenne sur des intervalles de temps d' $1 \mathrm{~h}$ permet de lisser de façon très satisfaisante les courbes de $R_{n}$ par rapport au temps.

Les flux de chaleur sensible sont mesurés à 17 et $2,7 \mathrm{~m}$ au-dessus du sol par 2 anémo- 
mètres soniques monodimensionnels CA27T (Campbell Scientific, Inc, Logan, Utah, USA) couplés avec des thermocouples fins chromelconstantan (Campbell et Unsworth, 1979; Biltoft et Gaynor, 1987). La fréquence supérieure de coupure du système est de $10 \mathrm{~Hz}$. Afin d'éviter une dérive de la moyenne de la composante verticale du vent et de la température de l'air, c'est-à-dire du 0 de $w^{\prime}$ et $T$, le terme $w^{\prime} T$ est moyenné sur $5 \mathrm{~min}$, les valeurs obtenues étant ensuite sommées, si nécessaire, sur des pas de temps plus longs.

A $18 \mathrm{~m}$ de haut, la couche limite de la forêt est supposée suffisamment homogène pour qu'un seul site de mesure de $H$ soit suffisant. A $2,7 \mathrm{~m}$, nous avons montre, en plaçant plusieurs capteurs dans le même plan horizontal à différentes distances des troncs, que la position de ceux-ci avait peu d'influence sur la valeur de $H$ (Diawara et al, 1991).

Les mesures sont effectuées de $6 \mathrm{~h}$ à $17 \mathrm{~h}$ TU environ, les anémomètres soniques, sensibles à l'eau, ne pouvant être maintenus en place la nuit. Dix-neuf journées de mesure des flux de chaleur latente, dont 11 avec l'ensemble des mesures microclimatiques, ont été retenues.

L'acquisition des données est faite sur 2 centrales de mesure $21 X$ et 2 centrales CR7X (Campbell scientific, Inc), les 2 premières utilisées pour les anémomètres soniques (fréquence d'acquisition $10 \mathrm{~Hz}$ ) et les 2 autres pour les mesures climatiques (fréquence d'acquisition $0,1 \mathrm{~Hz}$ ). En outre, les données présentant une inertie importante (température dans le sol et dans le bois) et ne nécessitant donc pas une grande fréquence d'acquisition, sont transmises à la centrale à travers 2 multiplexeurs AM32 (Campbell Scientific, Inc) afin d'économiser les voies d'entrée.

Le stock d'eau du sol est mesuré à l'aide d'une sonde à neutrons Solo 25 (Nardeux Humisol, Loches). L'étalonnage de la sonde est opéré sur 20 tubes d'accès au moment de la mise en place de ceux-ci : il consiste à mettre en relation l'humidité volumique de chaque strate, déterminée à partir de son humidité pondérale (pesage des échantillons avant et après

\section{Humidité volumique}

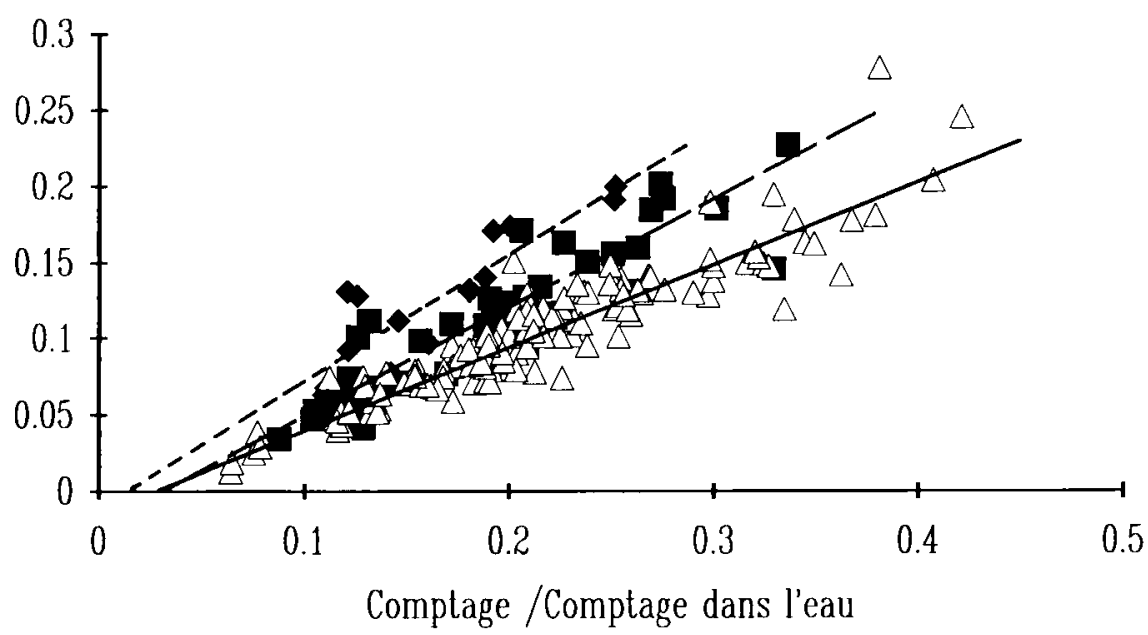

Fig 2. Étalonnage de la sonde à neutrons : humidité volumique en fonction du comptage rapporté au comptage dans l'eau; losanges pleins et droite pointillée : mesures à $10 \mathrm{~cm}$ de profondeur. $Y=0,8158 X-0,0083$; nombre d'individus $n=15$; coefficient de détermination $R^{2}=0,77$; carrés pleins et droite tiretée : mesures entre 15 et $35 \mathrm{~cm}$ de profondeur. $Y=0,7000 X-0,0224 ; n=45$; $R^{2}=0,84$; triangles ouverts et droite continue : mesures entre 45 et $135 \mathrm{~cm}$ de profondeur. $Y=0,541$ $7 X-0,0122 ; n=131 ; R^{2}=0,85$. 
séchage) et de sa densité apparente humide (gammamétrie), avec le résultat du comptage, rapporté à la valeur de référence obtenue dans l'eau. Les mesures sont faites à $10 \mathrm{~cm}$ de profondeur, puis tous les $10 \mathrm{~cm}$ de 25 à $135 \mathrm{~cm}$. Trois relations linéaires correspondant aux $\mathrm{ni}$ veaux $10 \mathrm{~cm}, 15-35 \mathrm{~cm}$ et $45-135 \mathrm{~cm}$ sont établies (fig 2).

Le stock hydrique est ensuite estimé par la moyenne arithmétique de 8 tubes d'accès permanent choisis parmi les précédents à partir de l'étude de la variabilité spatiale de l'humidité pondérale à 20 et $40 \mathrm{~cm}$ de profondeur, suivant une configuration optimisée par krigeage (Brejon de Lavergnée, 1988).

La profondeur maximale de sol utile estimée à $0,75 \mathrm{~cm}$, c'est-à-dire sensiblement jusqu'à l'alios. Dans ces conditions, le stock d'eau maximal au printemps, sur sol réessuyé, est de $140 \mathrm{~mm}$; le stock minimal observé en 1989 , année très sèche, étant de $31 \mathrm{~mm}$, la réserve utile est d'environ $110 \mathrm{~mm}$ (Loustau et al, 1990).

Entre les journées de mesure, le stock est estimé à partir de la dernière mesure neutronique et d'estimations du bilan hydrique de la forêt (Cochard, 1988).

\section{Résistances au flux de chaleur latente}

Terminologie. Nous utilisons les termes "résistance stomatique" ef "conductance stomatique" pour définir la résistance (ou conductance) moyenne de l'ensemble des stomates de chaque couche, et les termes arésistance de surface" et "conductance de surface" pour la résistance (conductance) moyenne de l'ensemble stomates plus couche limite des feuilles, ces résistances ou conductances étant rapportées à l'unité de surface de sol. Pour le houppier, où les résistances de couche limite des aiguilles sont négligeables devant les résistances stomatiques, ces 2 notions se confondent.

Les valeur des températures et pressions de vapeur variant très peu avec la hauteur dans le couvert, les résistances aérodynamiques du couvert se réduisent dans la pratique aux résistances moyennes des couches limites des feuilles de chaque strate.

Pour calculer les résistances au flux de chaleur latente, nous avons utilisé les mesures ef- fectuées à l'aide de psychromètres à 2 et $4 \mathrm{~m}$ du sol d'une part, à 8, 10,5 et $13 \mathrm{~m}$ d'autre part : celles-ci nous ont permis de calculer la pression de vapeur au-dessus du sous-bois (2 et $4 \mathrm{~m}$ ) et au niveau du houppier $(8,10,5$ et $13 \mathrm{~m})$. Par ailleurs, nous avons enregistré en continu, avec 10 thermocouples fins au contact de la face inférieure de feuilles de molinie, la température superficielle de celles-ci, et, à plusieurs occasions, avec un radiothermomètre, la température radiative moyenne des aiguilles de pin. En supposant que, d'une part, la température profonde des feuilles et aiguilles est égale en tous points à la température de surface, et que, d'autre part, l'air au contact de la feuille est saturé au niveau des cavités sous-stomatiques, nous pouvons écrire, pour chacun des 2 niveaux :

$$
r_{s}+r_{a}=\left[\rho C_{p} /(\gamma L E)\right]\left(e\left(T_{s}\right)-\theta_{a}\right)
$$

$r_{s}$ : résistance stomatique moyenne de la strate, rapportée à l'unité de surface du sol $\left(\mathrm{s} . \mathrm{m}^{-1}\right)$

$r_{a}$ : résistance aérodynamique au transfert de vapeur (moyenne de la strate) de la surface des feuilles ou des aiguilles $\left(\mathrm{s} \cdot \mathrm{m}^{-1}\right)$

$e_{s}\left(T_{s}\right)$ : pression partielle de vapeur d'eau saturante à la température $T_{s}$ de surface de la feuille $\left(P_{a}\right)$

$e_{a}$ : pression partielle de vapeur d'eau dans l'air $\left(P_{\mathrm{a}}\right)$

$\gamma$ : constante psychrométrique $\left(\approx 66 \mathrm{~Pa}^{\circ} \mathrm{C}^{-1}\right)$.

La résistance $r_{a}$, pour la convection laminaire (qui, compte tenu de la dimension des fevilles et des aiguilles, règne à leur surface, Monteith, 1973), peut se calculer à partir de l'expression de la résistance au flux de chaleur sensible dont elle ne diffère, en première approximation, que par une constante :

$$
r_{a}=\left[\rho C_{\rho} /(1,13 \mathrm{H})\right]\left(T_{s}-T_{a}\right)
$$

$T_{s}$ : température de surface des feuilles $\left({ }^{\circ} \mathrm{C}\right)$

$T_{a}$ : température de l'air $\left({ }^{\circ} \mathrm{C}\right)$

Le terme 1,13 est la valeur numérique approchée de la fonction de Le (nombre de Lewis, rapport de la diffusivité de l'air pour la vapeur d'eau à celle pour la chaleur), qui est, en convection laminaire, le rapport des résistances de la couche limite relatives respectivement aux flux de chaleur sensible et latente. La plupart des auteurs négligent ce terme correctif. 


\section{Résistances du houppier}

Amadou (1989) a montré que la résistance $r_{a h}$ de la couche limite des aiguilles du houppier, calculée par (5), est très faible (moins de $10 \mathrm{~s} . \mathrm{m}^{-1}$ ), et que de plus, les températures de surface des aiguilles sont pratiquement égales à celles de l'air au même niveau (moins de $1^{\circ}$ d'écart), ce qui justifie l'approximation courante chez les conifères (McNaughton et Black, 1973; Jarvis et Stewart, 1973; Jarvis, 1981) :

$$
r_{s h} \approx\left(\rho C_{p} \gamma\right)\left(D_{h} / L E_{h}\right)
$$

Les paramètres indicés avec $h$ ont la même signification que dans l'équation (4), mais sont pris au niveau du houppier. $L E_{h}$ est la part du flux de chaleur latente émise par la houppier.

$D=e_{s}\left(T_{a}\right)-e_{a}$ : déficit de saturation (Pa)

$\theta_{s}\left(T_{a}\right)$ : pression de vapeur saturante à la température ambiante $T_{a}(\mathrm{~Pa})$

$\theta_{a}$ : pression de vapeur dans l'air $(\mathrm{Pa})$.

\section{Résistances du sous-bois}

Amadou (1989) montre que la résistance $r_{a m}$ de la molinie (équation 5) n'est pas toujours négligeable devant sa résistance stomatique $r_{s m}$ : en effet, les valeurs obtenues sont de l'ordre de 40 a 100 s.m-1, contre des valeurs de $r_{\mathrm{sm}}$ qui peuvent être inférieures à $100 \mathrm{~s} \cdot \mathrm{m}^{-1}$. Toutefois ces dernières, qui ne sont observées que jusqu'à $10 \mathrm{~h}$ TU le matin, sont peut-être dues à la présence de rosée sur les feuilles.

Une forme équivalente de l'équation (4), appliquée à la végétation du sous-bois, peut s'écrire :

$r_{s m}+r_{a m}=\frac{\rho C_{p} D_{m}}{\gamma L E_{m}}\left[1+\frac{\left(\Theta\left(T_{s m}\right)-\Theta\left(T_{a m}\right)\right)}{D_{m}}\right]$

Les paramètres indicés par $m$ sont relatifs à la molinie.

II n'est possible, contrairement au cas du houppier, de négliger ni $r_{a m}$ ni $\left(e\left(T_{s m}\right)-e\right.$ $\left.\left(T_{a m}\right)\right) / D_{m}$ En effet, les écarts de température entre les feuilles de molinie et l'air peuvent atteindre $6^{\circ} \mathrm{C}$ en milieu de journée. Lorsqu'on né- glige ce dernier terme, le calcul montre que la résistance de couvert peut être surestimée le matin et le soir de plus de $30 \%$ (ce qui n'est pas très grave, car les échanges sont faibles), mais surtout sous-estimée de plus de $30 \%$ en milieu de journée, lorsque l'évapotranspiration est maximale.

Toutefois, afin de nous affranchir de la mesure permanente de la temperature de surface de la molinie, nous avons essayé, par analogie avec (6), de définir une résistance "stomatique approchée» $r_{s m}$ "de la molinie par l'équation :

$$
r_{s m}^{*}=\frac{\rho C_{p} D_{m}}{\gamma L E_{m}}
$$

La comparaison de cette résistance avec la résistance stomatique obtenue par le calcul complet (équations (4) et (5)) montre que ces 2 paramètres ne sont égaux qu'entre 100 et 300 s. $\mathrm{m}^{-1}$ environ (fig 1), zone où, dans l'équation (7), le fait de négliger $\mathrm{r}_{\mathrm{am}}$ est sensiblement compensé par l'approximation $T_{s m}=T_{a m}$. Toutefois, nous verrons par la suite qu'en l'absence de stress hydrique, la modélisation de $r_{\mathrm{sm}}{ }^{*}$, même si son sens physique est discutable, permet une très bonne prévision de $L E_{m}$.

L'approche par les résistances, si elle est intéressante du fait de l'additivité des résistances en série, est malcommode car celles-ci varient en sens inverse des flux. En particulier, une faible résistance de surface, estimée avec une forte erreur relative, correspond à un flux élevé : la précision de l'estimation de ce dernier à partir de la première est alors faible. C'est pourquoi, plutôt que $r_{s h}$ et $r_{s m}{ }^{*}$, nous utilisons par la suite leurs inverses, les conductances stomatiques $g_{s h}$ et $g_{s m}{ }^{*}\left(\mathrm{~m} \cdot \mathrm{s}^{-1}\right)$.

\section{Rapport LE/R}

A courte échelle de temps, lorsque les paramètres du microclimat peuvent être considérés comme constants, la conductance stomatique est facile à définir. A l'échelle de la journée, le fait de faire une moyenne journalière de la conductance stomatique $g_{s}: 1 / r_{s}$ conduit à donner le même poids à des valeurs correspondant à des évaporations très faibles (début et fin de journée) et très fortes (milieu de journée). 
Le rapport des valeurs, cumulées sur la journée, de $L E$ et de $R_{n}$ ne présente pas cet inconvénient. II représente la part du rayonnement net absorbé au niveau d'une strate qui est utilisée pour l'évaporation, donc rend bien compte à la régulation effectuée par la plante. En outre, à l'échelle horaire, si l'on admet les approximations des équations (5) et (8), en appelant $R_{n}$ le rayonnement absorbé par l'une ou l'autre des 2 strates, on montre que :

$$
\frac{L E}{R_{n}}=\frac{\rho C_{\rho}}{\gamma}\left[\frac{D g_{s}}{R_{n}}\right]
$$

Lohammar et al (1980) montrent qu'en l'absence de stress hydrique, la résistance stomatique d'un couvert de résineux, pris dans son ensemble, varie comme $\left\{\left(R_{g}+R_{0}\right) / R_{g}\right\}(D+a)$, où $A_{g}$ et $D$ sont respectivement le rayonnement global et le déficit de saturation au-dessus des arbres, $R_{0}$ et a des constantes d'ajustement. Nous avons essayé d'ajuster $r_{s h}$ et $r_{s m}{ }^{*}$ à ce modèle, modifié en remplaçant le rayonnement global par le rayonnement net absorbé à chacun des 2 niveaux $\left(R_{n h}\right.$ et $\left.R_{n m}\right)$, qui peut s'écrire :

$$
\begin{aligned}
& r_{s}=\left(1+a_{0} / R_{n}\right)\left(a_{1}+a_{2} D\right) \\
& =a_{1}+a_{1} a_{0} / R_{n}+a_{2} D+a_{2} a_{0} D / R_{n} \\
& a_{0}, a_{1}, a_{2} \text { : coefficients d'ajustement }
\end{aligned}
$$

Comme le montre la deuxième formulation de (10), le modèle peut être ajusté à l'aide d'une régression multiple sur les variables $1 / R_{n}, D$ et $D / R_{n}$, alors que l'étude de la conductance nécessite le recours à la régression non-linéaire.

Nous verrons plus loins que cette forme d'équation peut être simplifiée pratiquement sans perte de signification, dans les limites de variation de $R_{n}$ et $D$ de l'expérience présente, en :

$$
r_{s}=\left(\rho C_{\rho} r_{0} \cdot D\right) /\left(\gamma R_{n}\right)
$$

$r_{0}$ : constante d'ajustement adimensionnée

$r_{s}: r_{s h}$ ou $r_{s m}$ (équations 6 et 8)

Avec cette approximation, $L E / R_{n}=1 / r_{0}=g_{0}$ peut être considéré comme une expression adimensionnée de la conductance stomatique (équation 9), intégrant l'effet du microclimat.

\section{RÉSULTATS}

\section{Rayonnement net et transpiration}

Exprimé en équivalent du flux de vapeur (en $\mathrm{mm}$ d'eau), le rayonnement net cumulé sur la journée (en général sur $10 \mathrm{~h}$ de mesures) varie de 8,7 à $4,7 \mathrm{~mm}$ du solstice (jour $J_{163}$ à la fin des expériences (jour $\mathrm{J}_{269}$ ). La proportion de ce flux transmise au sol décroît avec la hauteur du soleil, passant de 37 à $21 \%$ dans la même période (respectivement 3,18 et $0,98 \mathrm{~mm}$ ).

La transpiration du houppier représente, en début d'expérience $\left(J_{138}-J_{174}\right)$, de 50 à $60 \%$ du rayonnement net absorbé par cette couche; après dessèchement du sol $\left(J_{184}-J_{237}\right)$, cette proportion fluctue entre 26 et $42 \%$, puis tombe à $11-17 \%$ en fin de saison végétative $\left(J_{263}-J_{269}\right)$. Les ordres de grandeur des transpirations correspondantes sont respectivement $2,7 \mathrm{~mm}$, $1,5 \mathrm{~mm}$ et $0,6 \mathrm{~mm}$ par jour.

La transpiration de la molinie représente une proportion plus grande du rayonnement net absorbé à son niveau : après une phase d'installation du couvert en mai, la proportion culmine à $89 \%$ à $J_{145}$, puis décroît sans changements brusques jusqu'à $J_{184}$ où elle atteint $63 \%$; elle se maintient ensuite entre 60 et $66 \%$ jusqu'à $J_{237}$, puis, profitant, contrairement aux pins, des pluies de fin de saison, augmente pour atteindre $72 \%$ à $J_{269}$. Les valeurs typiques de la transpiration correspondant aux périodes décrites pour les pins sont $2,2 \mathrm{~mm}$ par jour de $\mathrm{J}_{138}$ à $\mathrm{J}_{174}$ (c'est-à-dire $45 \%$ de l'évaporation totale), 1,2 $\mathrm{mm}$ de $\mathrm{J}_{184}$ à $\mathrm{J}_{237}$ (45\%) et $0,7 \mathrm{~mm}$ de $J_{263}$ à $J_{269}(54 \%)$. La molinie représente donc une part très importante de l'évapotranspiration totale. 


\section{Ordres de grandeur des conductances stomatiques}

Faute de données d'humidité de l'air, les conductances stomatiques du houppier n'ont été déterminées qu'à partir du jour 163 (12 juin). Quant à la molinie, les températures de feuilles, donc $g_{s m}$, ne sont disponibles qu'à partir du jour 170 (19 juin).

La figure 3 montre l'évolution des conductances de surface moyennes du houppier et de la molinie pour 2 journées caractéristiques, le jour 170 (19 juin) en l'absence de déficit hydrique, et le jour 263 (20 septembre), avec un déficit hydrique accentué. Nous présentons, pour la molinie, les valeurs de la conductance de surface $g_{s a m}=1 /\left(r_{s m}+r_{a m}\right)$ calculées d'après l'équation (7), qui ne nécessité pas le calcul séparé (équation 5) de la résistance $r_{a m}$, qui est très imprécis en début et en fin de journée. La figure montre de façon très claire la fermeture stomatique des aiguilles de pin en fin de matinée et pendant tout l'après-midi en période de stress hydrique, alors qu'en début d'expérience, les variations de leur conductance stomatique durant la journée sont relativement faibles. La molinie est, elle aussi affectée, mais dans une moindre mesure. Les très fortes valeurs des conductances stomatiques observées en septembre (jour 263) en début de journée, sont probablement dues à l'évaporation de la rosée, très abondante en cette période (le déficit de saturation est très faible en début de matinée), qui court-circuite les résistances stomatiques.

Tableau I. Moyennes horaires pour l'ensemble des journées expérimentales des conductances de surface $\left(10^{-2} \mathrm{~m} \cdot \mathrm{s}^{-1}\right)$, des déficits de saturation $(\mathrm{Pa})$ et des rayonnements nets $\left(W \cdot \mathrm{m}^{-2}\right)$ absorbés au niveau du houppier et du sous-bois de molinie, le matin après dissipation de la rosée (entre 9 et $10 \mathrm{~h}$ TU) et le soir entre 15 et $16 \mathrm{~h}$ TU. Les jours 163 et 164, les températures des feuilles de molinie n'étaient pas mesurées. Le jour 220, les mesures n'ont commencé qu'à $10 \mathrm{~h}$ TU. Les indices $h$ font référence au houppier, $m$ a la molinie. $D$ : déficit de saturation de l'air au niveau de la strate végétale, $R_{n}$ : rayonnement net absorbé par celle-ci, $g_{s h}$ : conductance stomatique moyenne du houppier par $\mathrm{m}^{2}$ de sol, $g_{\text {sam }}$ : conductance de surface moyenne (stomates et couche limite) de la molinie par $\mathrm{m}^{2}$ de sol.

\begin{tabular}{|c|c|c|c|c|c|c|c|c|c|c|c|c|}
\hline \multirow[b]{3}{*}{$J$} & \multicolumn{6}{|c|}{ Houppier } & \multicolumn{6}{|c|}{ Sous-bois } \\
\hline & \multicolumn{3}{|c|}{$9-10 h$} & \multicolumn{3}{|c|}{$15-16 h$} & \multicolumn{3}{|c|}{$9-10 h$} & \multicolumn{3}{|c|}{$15-16 h$} \\
\hline & $D_{h}$ & $R_{n h}$ & $g_{s h}$ & $D_{h}$ & $R_{n h}$ & $g_{s h}$ & $D_{m}$ & $R_{n m}$ & $g_{s a m}$ & $D_{m}$ & $R_{n m}$ & $g_{\text {sam }}$ \\
\hline 163 & 1546 & 493 & 1,14 & 2745 & 344 & 0,44 & - & - & - & - & - & - \\
\hline 164 & 574 & 463 & 2,33 & 1229 & 355 & 0,81 & - & - & - & - & - & - \\
\hline 170 & 1585 & 466 & 0,93 & 3184 & 368 & 0,44 & 1545 & 108 & 0,30 & 2889 & 143 & 0,26 \\
\hline 171 & 1231 & 460 & 1,33 & 3008 & 336 & 0,40 & 1140 & 111 & 0,37 & 2739 & 120 & 0,24 \\
\hline 172 & 1400 & 292 & 0,56 & 3155 & 351 & 0,36 & 1894 & 112 & 0,27 & 2899 & 126 & 0,21 \\
\hline 174 & 1531 & 478 & 1,14 & 2531 & 375 & 0,48 & 1436 & 100 & 0,24 & 2287 & 137 & 0,26 \\
\hline 215 & 1771 & 365 & 0,40 & 2968 & 268 & 0,10 & 1800 & 138 & 0,16 & 2981 & 170 & 0,20 \\
\hline 220 & - & - & - & 1366 & 283 & 0,46 & - & - & - & 1244 & 138 & 0,42 \\
\hline 236 & 1049 & 279 & 0,65 & 2365 & 225 & 0,12 & 1011 & 141 & 0,49 & 2226 & 94 & 0,18 \\
\hline 237 & 1600 & 305 & 0,50 & 2449 & 300 & 0,19 & 1523 & 147 & 0,34 & 2309 & 94 & 0,15 \\
\hline 263 & 629 & 336 & 0,88 & 1897 & 227 & 0,09 & 613 & 57 & 0,25 & 1786 & 43 & 0,14 \\
\hline 264 & 807 & 322 & 0,30 & 2113 & 214 & 0,03 & 781 & 56 & 0,23 & 2107 & 41 & 0,13 \\
\hline 269 & 892 & 309 & 0,33 & 1686 & 211 & 0,23 & 810 & 55 & 0,28 & 1593 & 27 & 0,12 \\
\hline
\end{tabular}


Le tableau I donne, pour toutes les journées expérimentales, les valeurs de la conductance stomatique au niveau du houppier et de la molinie le matin entre 9 et $10 \mathrm{~h}$ TU (heure à laquelle la rosée a en général disparu) et le soir entre 15 et $16 \mathrm{~h}$ TU (avant que le rayonnement ne devienne trop faible sous le couvert), ainsi que les valeurs du déficit de saturation et du rayonnement global correspondantes. II confirme les conclusions tirées de la figure 3. Le début de la forte fermeture stomatique l'après-midi (probablement liée au stress hydrique) semble se situer après le jour 174. La réaction de la molinie est plus faible et plus progressive.

\section{Effet des parametres climatiques sur les résistances stomatiques}

Compte tenu des remarques précédentes, nous avons systématiquement éliminé les mesures faites avant $8 \mathrm{~h}$ TU, qui, sans doute par suite de la présence de rosée, donnaient des valeurs de résistance de surface très faibles, parfois nulles.

Pour les jours compris entre 163 et 174 (pas de stress hydrique), le modèle décrit par l'équation (10) semble ne rendre compte qu'assez grossièrement de la résistance du houppier (coefficient de détermination $R^{2}=0,55$, nombre d'heures de mesure $n=55$ ). Il est intéressant de noter
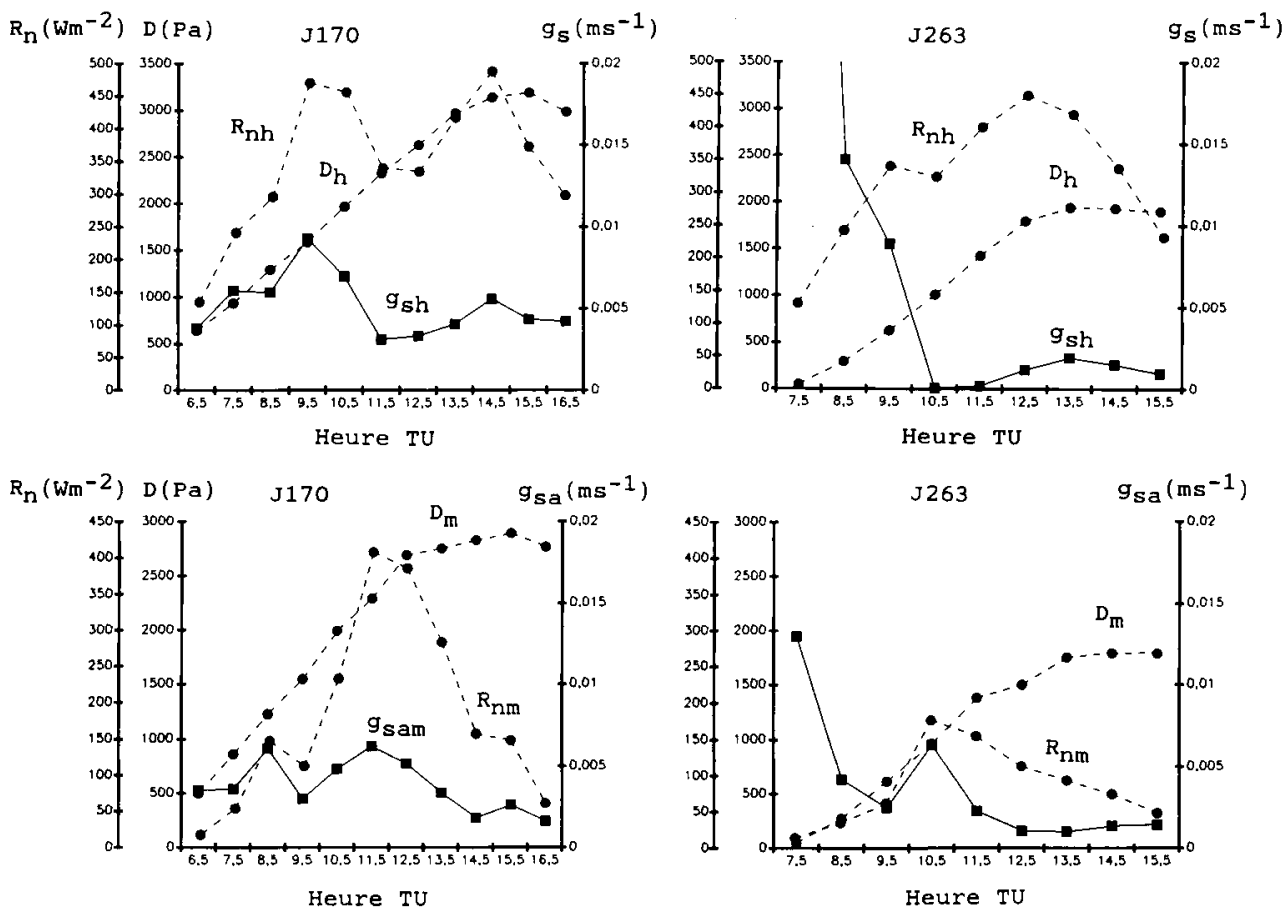

Fig 3. Evolution horaire de la conductance stomatique moyenne du houppier et de la conductance de surface (stomates et couche limite des feuilles) moyenne de la molinie en début ( $J_{170}: 19$ juin) et en fin $\left(J_{263}: 20\right.$ septembre) de la période de sècheresse, ainsi que du déficit de saturation $D$ et du rayonnement net $R_{n}$. Les indices $h$ sont relatifs au houppier, les indices $m$ à la molinie. 
que la régression simple sur $D / R_{n}$ donne un ajustement qui n'est pas statistiquement inférieur au précédent $\left(R^{2}=0,52\right.$, différence non significative au seuil de probabilité $5 \%$ ).

Pour la molinie (jours 170-174), l'ajustement à partir de l'équation (10) est meilleur $\left(R^{2}=0,74, n=36\right)$. Là aussi, la régression simple sur $D / R_{n}$ donne d'aussi bons résultats $\left(R^{2}=0,70\right.$, différence non significative).

Nous avons donc choisi d'utiliser la régression simple dans les 2 cas, et de faire porter l'analyse sur les conductivités, pour lequelles ne se pose plus alors le problème de la non-linéarité.

Le tableau II donne les paramètres des régressions simples de $g_{s h}$ et $g_{s m}$ "en fonction de $R_{n} / D$ pris respectivement au niveau du houppier et de la molinie, pour les jours précédant le jour 174. De façon générale, les coefficients de détermination sont meilleurs que pour les résistances, ce qui s'explique par le fait que, contrairement à ces dernières, les valeurs présentant la plus grande incertitude (valeurs les plus faibles des flux) sont les plus basses et ont le moins de poids dans le calcul des régressions.

L'ajustement de $g_{s m}$ " est particulièrement bon ( $R^{2}=0,98$; fig 4). La droite de régression passe pratiquement par l'origine; par ailleurs, l'effet du dessèchement du sol sur $g_{s m}{ }^{*}$ est faible. Comme $g_{s m}{ }^{*}$ n'est qu'une valeur approchée de la conductance de surface de la molinie, if est préférable, pour juger de la valeur de l'ajustement, de comparer les valeurs de l'évaporation, estimées par $\rho C_{p} / \gamma g_{s m}{ }^{*} D_{m}$ $=0,781 R_{n m}$, aux valeurs mesurées : la figure 5 montre que la concordance est très bonne avant le jour 174 , et que l'effet ultérieur du dessèchement du sol est réduit.

En revanche, le tableau II et la figure 6 montrent que l'ajustement de $g_{s h}$ est moins bon $\left(R^{2}=0,83\right)$.

Le forçage de la régression par l'origine diminue notablement le coefficient de détermination $\left(R^{2}=0,68\right)$. Cependant, l'examen de la figure 6 montre que la différence est essentiellement due à un point de conductance très élevée $\left(g_{\text {sh }}=2,33\right.$

Tableau II. Jours précédant l'apparition du stress hydrique (jusqu'au jour 174) : coefficients des ré-

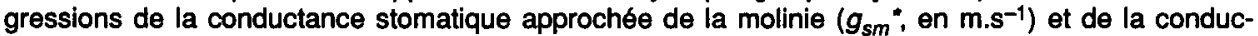

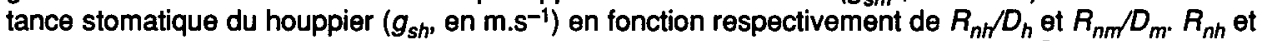
$R_{n m}$ : rayonnements nets absorbés au niveau du houppier et de la molinie en W. $\mathrm{m}^{-2}, D_{h}$ et $D_{m}$ : déficits de saturation aux mêmes niveaux en $\mathrm{Pa}, n$ : nombre d'heures de mesure.

\begin{tabular}{lccc}
\hline & $\begin{array}{c}\text { Coefficient } \\
\text { de } \\
\text { régression }\end{array}$ & $\begin{array}{c}\text { Ordonnées } \\
\text { à } \\
\text { I'origine }\end{array}$ & $R^{2}$ \\
\hline Molinie $(\mathrm{n}=36)$ & & & \\
$\begin{array}{l}\text { Régression simple } \\
\text { Droite forcée par l'origine }\end{array}$ & $4,36 \times 10^{-2}$ & $-6,47 \times 10^{-6}$ & 0,978 \\
Houppier $(\mathrm{n}=54)$ & $4,36 \times 10^{-2}$ & 0 & 0,978 \\
$\begin{array}{l}\text { Régression simple } \\
\text { Droite forcée par l'origine }\end{array}$ & $3,57 \times 10^{-2}$ & $3,77 \times 10^{-3}$ & 0,827 \\
\hline
\end{tabular}




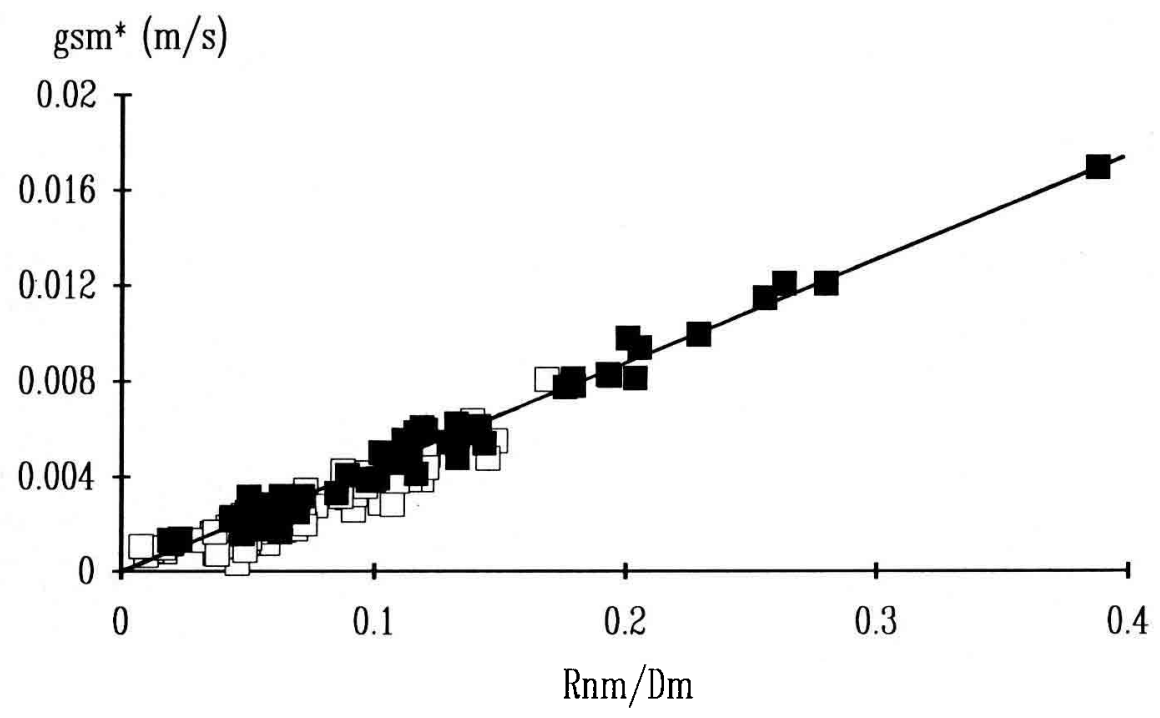

Fig 4. Relation entre le rapport des moyennes horaires du rayonnement net au déficit de saturation au niveau du sous-bois, et la valeur horaire de la conductance stomatique approchée $g_{s m}{ }^{*}=1 / r_{s m}{ }^{*}$ (équation 8); symboles pleins : jusqu'au jour 174; symboles ouverts : après le jour 174; en traits pleins, droite de régression forcée par l'origine calculée jusqu'au jour $174: Y=0,0436 X ; n=36$; $R^{2}=0,98$

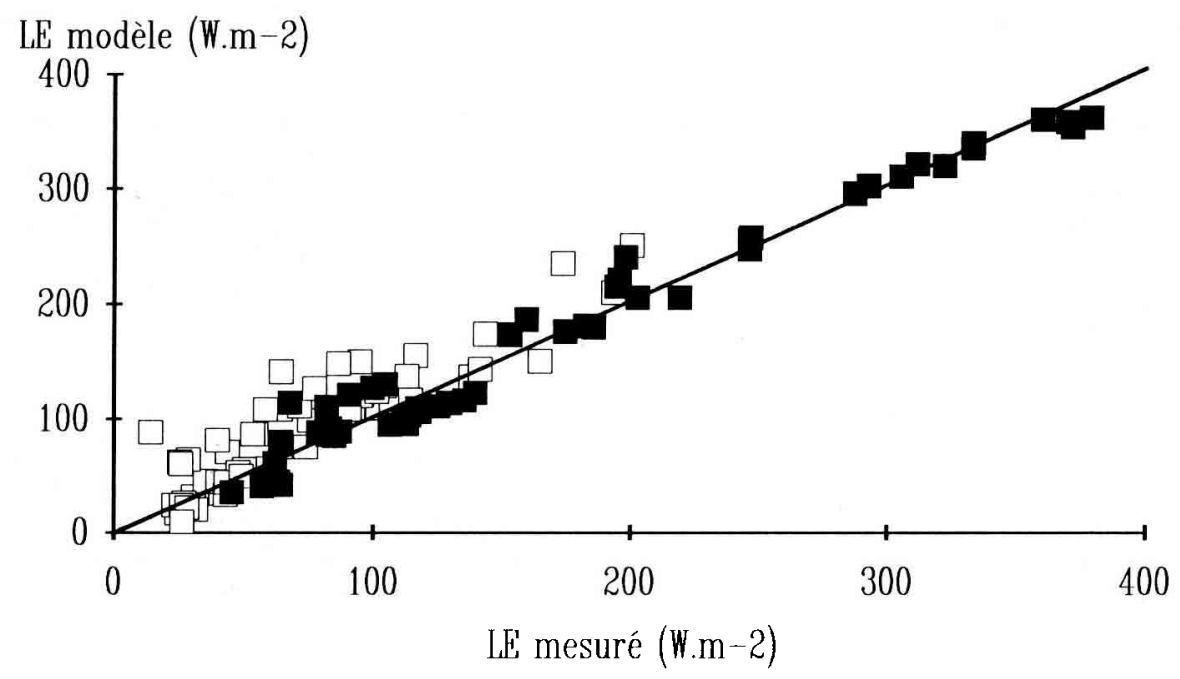

Fig 5. Relation entre le flux de chaleur latente du sous-bois estimé par la régression de la figure 4 et la valeur mesurée. Symboles pleins : jusqu'au jour 174. Symboles ouverts : après le jour 174. En traits pleins : droite $Y=X$. 


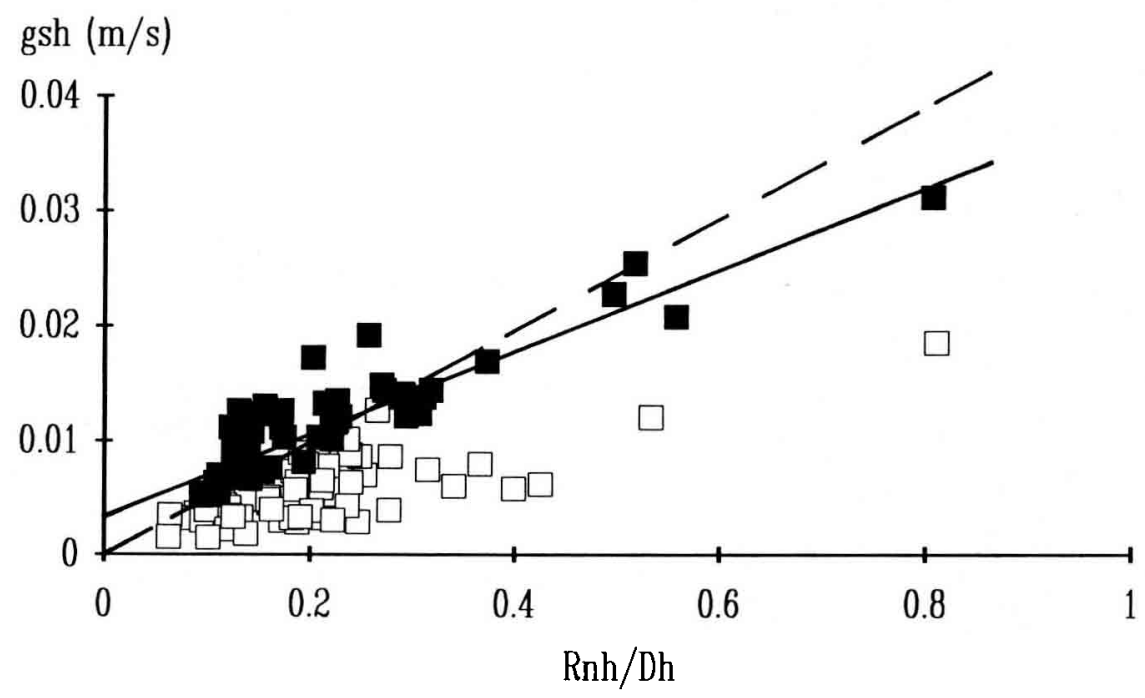

Fig 6. Relation entre le rapport des moyennes horaires du rayonnement net absorbé et du déficit de saturation au niveau du houppier, et la valeur horaire de la conductance stomatique moyenne $g_{s h}=1$ / $r_{s h}$ (équation 6); symboles pleins : jusqu'au jour 174; symboles ouverts : après le jour 174; en traits pleins, droite de régression calculée jusqu'au jour $174: Y=0,0357 X+0,00377 ; n=54 ; R^{2}=0,83$; en tiretés, droite forcée par l'origine : $Y=0,0485 X ; n=54 ; R^{2}=0,68$.

$\left.m . s^{-1}\right)$, correspondant au jour 164 entre 9 et $10 \mathrm{~h}$ TU, heure à laquelle la rosée n'était peut-être pas évaporée. Ce point mis à part, le forçage par l'origine n'altère pas sensiblement la qualité de l'ajustement.

Comme nous l'avions déjà vu sur le tableau 1, le dessèchement du sol après le jour 174 provoque une forte fermeture stomatique.

\section{$\mathrm{LE} / \mathbf{R}_{\mathrm{n}}$ à l'échelle de la journée}

Sur la figure 7 , nous avons porté le rapport des valeurs cumulées sur la durée de fonctionnement des anémomètres soniques (en général de 6 h à 17 h TU) de l'évaporation de chaque strate par le rayonnement net absorbé par celle-ci. Au niveau du houppier, ce rapport est également estimé à partir des valeurs horaires du flux de sève, cumulé sur le même intervalle. Nous avons aussi porté les valeurs du stock d'eau du sol de la surface à l'alios, mesuré ou interpolé à partir du bilan hydrique.

L'évolution de $L E / R_{n}$ a déjà été décrite : les figures 7 et 8 illustrent la dépendance de ce paramètre sur le stock en eau du sol et la pluie. Pour le houppier, ce rapport, calculé à partir du bilan d'énergie, ne commence à décroître que lorsque le stock en eau a déjà diminué de plus de moitié, entre les jours $J_{174}$ ( $37 \%$ du stock initial) et $184(24 \%)$. II chute alors brusquement, puis fluctue ensuite jusqu'à $J_{237}$, pour diminuer encore en fin d'expérience. La figure 


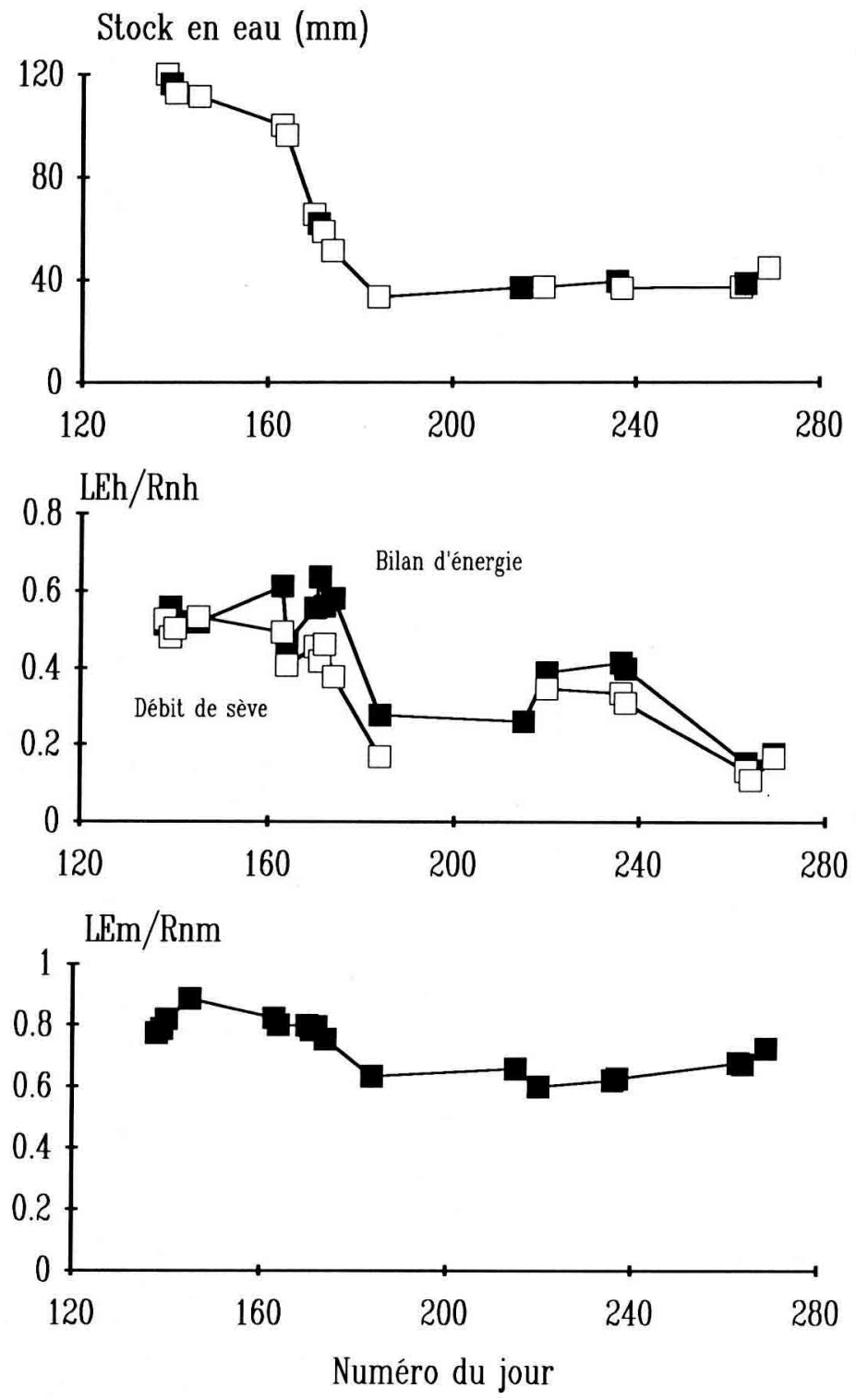

Fig 7. Évolution du stock en eau entre 0 et $75 \mathrm{~cm}$ dans le sol (symboles pleins : valeurs mesurées, symboles ouverts : interpolations), du rapport $L E_{h} / R_{n h}$ au niveau du houppier, évalué à l'aide du flux de sève (symboles ouverts) et du bilan d'énergie (symboles pleins), et du rapport $L E_{m} / R_{n m}$ au niveau de la molinie, pour toutes les journées expérimentales. Les valeurs de $L E / R_{n}$ sont les rapports des cumuls journaliers de $L E$ et $R_{n}$. 
8, qui représente les variations de $L E_{h} / R_{n h}$ en fonction des pluies des 6 jours précédents (paramètre que nous avons choisi pour des raisons de commodité de démonstration : nous ne prétendons pas qu'il est le plus pertinent), montre que, de $J_{215}$ à $\mathrm{J}_{237}$, ce rapport est étroitement lié aux pluies. En fin de saison, la chute de l'évapotranspiration est sans doute liée à l'entrée en dormance des arbres.

L'utilisation de la méthode du débit de sève conduit à des valeurs de $L E / R_{n}$ qui peuvent être inférieures de 0,1 au maximum à celles données par la méthode micrométéorolgique.

Pour le sous-bois, le rapport $L E_{m} / R_{n m}$ est, comme nous l'avons déjà vu, plus élevé et moins variable que pour les pins. L'effet du dessèchement du sol est peu accentué et celui des pluies n'apparaît pas sur nos données.

\section{DISCUSSION}

\section{Critique des méthodes}

La base de cette analyse est la mesure des différentes voies d'échanges thermiques par une méthode combinant le bilan d'énergie classique (équations 2 et 3 ) avec la mesure des covariances turbulentes de la vitesse verticale de l'air et de sa température (équation 1). Cette méthode est appliquée à chacune des 2 strates de végétation (houppier et sous-bois). Au niveau du houppier, il est possible de comparer cette méthode à celle du flux de sève à l'échelle de la journée (fig 7; Diawara, 1990). Pour l'ensemble des journées présentées ici, la corrélation entre les 2 paramètres est bonne $\left(Y=0,754 X+0,094, R^{2}=0,87 ; Y\right.$ est le flux de sève, $X$ la transpiration), mais le bilan d'énergie donne en général des ré-

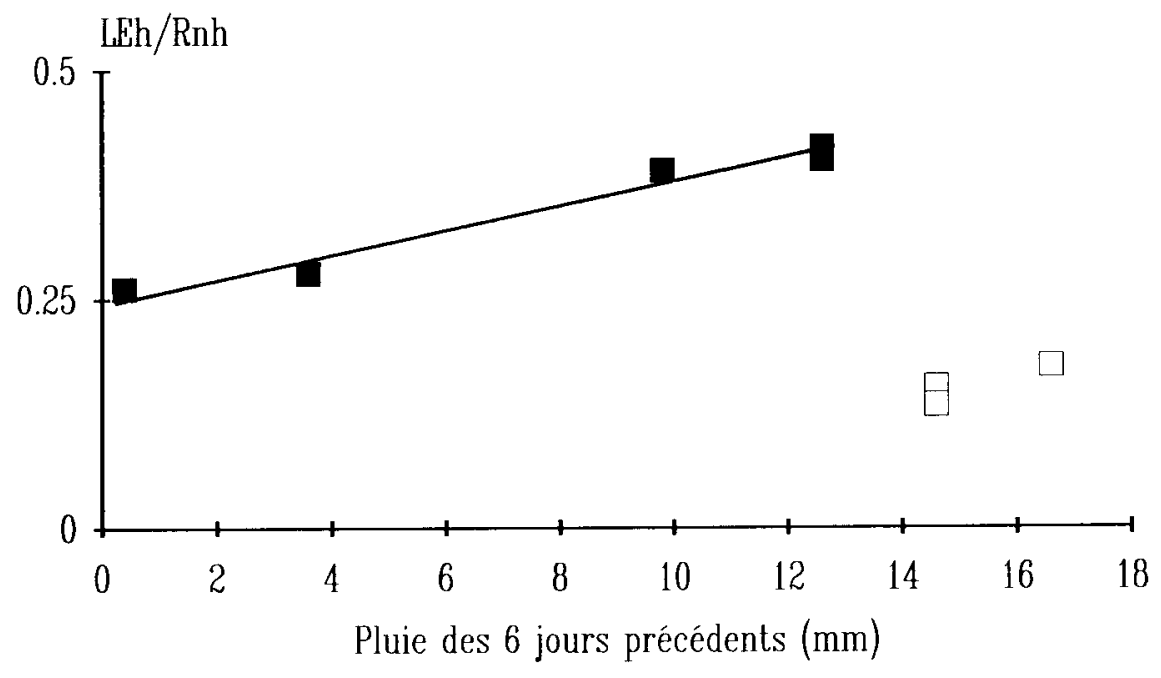

Fig 8. Évolution de $L E_{h} / R_{n h}$ journalier au niveau du houppier en fonction de la pluie des $6 \mathrm{j}$ précédents, après la fin du dessèchement de la couche $0-75 \mathrm{~cm}$ du sol. Symboles pleins : de $J_{185}$ à $J_{237}$. Symboles ouverts : de $J_{263}$ a $J_{269}$. 
sultats supérieurs au flux de sève, la différence correspondant à l'évaporation de 0 à $1,16 \mathrm{~mm}$ d'eau. L'évaporation de la rosée ou de la pluie pourrait expliquer cette différence. Par ailleurs, le flux de sève sous-estime l'évaporation le matin, car une partie de celle-ci est due à l'eau stockée dans le tronc pendant la nuit, et la surestime le soir, car une partie du flux de sève sert au stockage d'eau dans les troncs (Waring et al, 1979; Schulze et al, 1985). Seuls des cumuls sur 24 h du flux de sève et de l'évaporation peuvent donc, en toute rigueur, être comparés : or nos mesures se limitent à l'intervalle entre 6 et $17 \mathrm{~h}$ TU. II n'est pas possible, avec les données présentes, de séparer ces 2 effets : aussi ne pouvons-nous conclure sur leurs poids respectifs.

Nous n'avons malheureusement pas de possibilité de comparaison directe, au niveau du sous-bois, entre le bilan d'énergie et la cage à transpiration (El Hadj Moussa, 1989), qui permet d'isoler une touffe de molinie et d'en mesurer la transpiration à partir de l'élévation de la concentration de vapeur d'eau : en effet, nous n'avons pas pu effectuer de mesures simultanées.

L'estimation de la résistance stomatique à partir de méthodes micrométéorologiques est simplifiée, pour les conifères, par le fait que les résistances aérodynamiques sont négligeables par rapport à la résistance stomatique des aiguilles (Jarvis, 1980), ce qui permet d'estimer simplement cette dernière, ainsi que son inverse, la conductance stomatique, à partir du flux de chaleur latente et du déficit de saturation de l'air au-dessus ou à l'intérieur du couvert (équation 6). Nous avons, par ailleurs, vérifié la validité de cette approximation pour le pin maritime (Amadou, 1989). Pour le sous-bois de molinie, où la résistance aérodynamique de la végétation n'est pas négligeable devant la résis- tance stomatique (Amadou, 1989), nous définissons une résistance stomatique approchée par la même expression (équation 8). Bien que la valeur obtenue ne soit représentative de la résistance stomatique que dans un domaine étroit (fig 1), son utilisation, ou plutôt celle de son inverse, la conductance stomatique approchée, permet une excellente prévision de la transpiration du sous-bois en l'absence de stress hydrique (fig 4 et 5).

À l'échelle de la journée, la définition d'une résistance stomatique moyenne et son calcul à partir des paramètres microclimatiques moyens est malaisée. Or, le rapport de l'évapotranspiration de chaque strate au rayonnement net absorbé par celle-ci représente la part de l'énergie radiative captée qui est réémise sous forme évaporative : dans des conditions climatiques identiques, on comprend intuitivement que la diminution de ce rapport est directement liée à la fermeture des stomates. À l'échelle horaire, ce rapport est une fonction simple de la conductance stomatique moyenne, du rayonnement net absorbé et du déficit de saturation au niveau de la strate, sous réserve que les approximations permettant d'établir l'équation (9) soient valides. À l'échelle de la journée, il suffit de calculer le rapport des valeurs cumulées de l'évaporation et du rayonnement net : ce calcul ne privilégie pas, comme le ferait celui d'une conductance stomatique moyenne, les périodes de flux faibles pour lesquelles $g_{s}$ a le même poids que pour les périodes d'échanges énergétiques intenses.

\section{Ordres de grandeur des conductances}

Les seules données comparables sur le pin maritime sont issues de l'expérience Hapex-Mobilhy (André et al, 1986) sur la 
forêt des Landes, mais avec des arbres plus hauts $(20 \mathrm{~m})$, plus clairsemés (indice foliaire : 2,3 ), et avec un sous-bois de fougères : le flux de chaleur latente était mesuré directement à partir des fluctuations de la concentration de vapeur d'eau et de la vitesse verticale du vent (Shuttleworth et al, 1988). Gash et al (1989) en déduisent une conductance globale du couvert dont les valeurs maximales sont de l'ordre de $0,022 \mathrm{~m} . \mathrm{s}^{-1}$. Cette valeur est sensiblement égale à l'inverse de la somme des résistances du houppier et du sous-bois $\left(r_{s h}+\right.$ $\left.r_{\text {sam }}\right)^{-1}$ le matin, où elles sont, en général, maximales (valeurs moyennes entre 9 et $10 \mathrm{~h}$ TU des jours 170 à $174: 0,023 \mathrm{~m} . \mathrm{s}^{-1}$; tableau I). Sur le même site, avec la nappe phréatique proche de la surface du sol, les mesures porométriques de Granier et al (1990) sur les aiguilles de pin donnent à $9 \mathrm{~h}$ TU des maxima compris entre 0,002 et $0,003 \mathrm{~m} . \mathrm{s}^{-1}$ par rapport à la surface développée des aiguilles $\left(0,009\right.$ et $0,014 \mathrm{~m} . \mathrm{s}^{-1}$ par rapport à l'unité de surface du sol), alors que la moyenne entre 9 et $10 \mathrm{~h}$ des jours compris entre $J_{163}$ et $J_{174}$ est ici de 0,012 pour le houppier seul. Les valeurs de Granier et al correspondent à celles observées sur d'autres pins $\left(0,0034 \mathrm{~m} . \mathrm{s}^{-1}\right.$ par rapport à la surface développée pour Pinus sylvestris, Whitehead et al, 1984, 0,0032 pour Pinus resinosa, Waring, 1976). Bien que les couverts soient sensiblement différents (indice foliaire, sousbois), les ordres de grandeur de nos conductances stomatiques semblent donc réalistes.

À notre connaissance, il n'existe pas dans la littérature d'autres données sur la conductance stomatique de la molinie, que celles obtenues sur notre site expérimental.

\section{Modélisation de l'effet des paramètres climatiques}

II est évident que les relations linéaires entre les conductances stomatiques $g_{s h}$ et $g_{s m}{ }^{*}$ et le rapport $R_{n} / D$ (c'est-à-dire, si les droites de régression passent par l'origine, la proportionnalité du flux de chaleur latente et du rayonnement net, équations (6), (8) et (11)) en l'absence de stress hydrique ne sont réalistes que lorsque, ni $h_{n}$ ni $D$ ne dépassent une certaine valeur seuil : au-delà apparaît un phénomène de saturation et les conductances tendent vers une valeur constante. Les modèles semi-empiriques de conductance stomatique (Lohammar et al, 1980; Stewart, 1988; Shuttleworth, 1989) se présentent sous la forme d'un produit de fonctions de variables climatiques (essentiellement rayonnement global et déficit de saturation, parfois température du feuillage) et du déficit hydrique du sol, chacune de ces fonctions tendant vers une valeur constante pour les grandes valeurs du paramètre explicatif, sauf celle décrivant l'effet de la température, qui passe par un maximum. Tout le problème est de savoir si les valeurs de $R_{n}$ et de $D$ de l'expérience présente se situent ou non dans la partie linéaire des fonctions correspondantes.

Nous n'avons, bien sûr, aucune référence pour estimer l'étendue de cette zone pour la molonie : cependant, la très bonne linéarité de la relation $L E_{m}=0,781 R_{n m}$ (figs 4 et 5 ) permet de penser que les valeurs de $R_{n}$ dans le couvert y sont toutes comprises. Le rayonnement net arrivant au niveau du sol est relativement faible : sur l'ensemble des journées expérimentales, le maximum de la moyenne horaire de $R_{n m}$ est de 457 W.m-2 le 26 juin $\left(J_{163}\right)$. 
Sur l'ensemble du couvert, les données de Gash et al (1988) sur la forêt des Landes, semblent montrer que la relation entre la conductance de surface et le rayonnement global s'écarte de. la linéarité dès que ce dernier atteint $500 \mathrm{~W} \cdot \mathrm{m}^{-2}$ : selon la relation trouvée par ces auteurs $\left(R_{n t}=0,827 R_{g}-23,0\right)$, ce seuil correspond à un rayonnement net total de 390 $W \cdot m^{-2}$. Même si le comportement du sous-bois de fougère est inconnu, il est probable qu'une partie au moins de ce phénomène est dû aux pins. Or, le rayonnement net maximal (moyenne horaire) mesuré au-dessus du couvert dans l'expérience présente est de $770 \mathrm{~W} \cdot \mathrm{m}^{-2}$, très au-delà de la zone de linéarité.

En revanche, à partir des mêmes données, il est possible de montrer que l'effet négatif du déficit de saturation peut être ajusté à un modèle hyperbolique en $1 / D$ (ou, de manière presque aussi précise, à un modèle linéaire décroissant en $D$ ) de 400 jusqu'à au moins $2700 \mathrm{~Pa}$ : or $D$, dans l'expérience présente, ne dépasse pas $3200 \mathrm{~Pa}$.

Le dépassement du seuil de rayonnement net peut constituer une explication de la mauvaise linéarité de la relation entre $g_{s h}$ et $R_{n h}$ : cependant, jusqu'au jour 174 , pratiquement toutes les $h$ de mesure correspondent à un rayonnement net total supérieur à 390 W.m $\mathrm{m}^{-2}$ ( $8 \mathrm{~h}$ seulement où $R_{n t}$ est inférieur à ce seuil), et malgré celà, la non-linéarité n'est absolument pas évidente (fig 6). L'approximation linéaire n'est pas très rigoureuse, mais elle n'est pas mauvaise. Elle constitue un moyen commode de distinguer visuellement les journées où l'alimentation en eau est suffisante des journées de stress hydrique. En outre, complétée par l'hypothèse que la droite de régression passe par l'origine, elle permet de relier simplement la notion de conductance stomatique au rapport $L E /$ $R_{n}$. Des recherches ultérieures devraient permettre de préciser ses limites de validité.

\section{Étude du rapport $L E / R_{n}$}

Nous avons démontré (équation 11) que, sous réserve de l'hypothèse de proportionnalité de la conductance stomatique à $R_{r} / D$, ce rapport était, à l'échelle horaire, équivalent à la conductance stomatique adimensionnée $g_{0}$, aussi bien au niveau du houppier qu'à celui du sous-bois. $\mathrm{Ce}$ paramètre doit donc être indépendant du microclimat, et ne dépendre que du stress hydrique. Si celui-ci est décrit par la réserve en eau du sol, on ne dipose en pratique que de données journalières, ce qui nécessite l'utilisation du rapport des valeurs de $L E$ et $R_{n}$ cumulées sur la journée.

Shuttleworth (1989) décrit une réponse au dessèchement du sol conforme à nos résultats sur les pins (fig 7) : une réponse initiale faible, suivie d'une chute rapide de la transpiration vers des valeurs nulles lorsque la réserve facilement utilisable de la zone racinaire tend vers 0 . Pour des sols sableux, Shuttleworth donne, pour cette limite, des valeurs d'humidité volumique de $7 \%$.

Pour rendre compte de cette relation, Stewart (1988) propose une relation de la forme :

$$
g(\delta \theta)=1-\exp ^{\mathrm{K}\left(\delta \theta-\delta \theta_{\mathrm{m}}\right)}
$$

$\delta \theta$ : déficit d'humidité volumique du sol $\left(\mathrm{kg} \cdot \mathrm{m}^{-3}\right)$

$\delta \theta_{\mathrm{m}}$ : maximum de $\delta \theta$ déterminé empiriquement

$g(\delta \theta)$ : fonction décrivant l'effet de l'humidité du sol (ici, proportionnelle à $g_{0}$ ).

Nous n'avons pas un nombre de données suffisant pour ajuster les valeurs journalières de $\mathrm{LE}_{h} / \mathrm{R}_{n h}$ à ce type de mo- 
dèle : en effet, il ne décrit correctement que la période allant jusqu'à la fin de la fermeture des stomates, avant l'apparition de pluies $\left(\mathrm{J}_{220}\right)$. L'utilisation des données de flux de sève, beaucoup plus complètes, devrait nous permettre de le faire. Il en est de même pour les effets des pluies et de l'entrée en dormance des arbres (fig 8). II faudra probablement tenir compte, comme le fait Choisnel (1985), de 2 réservoirs, l'un profond (jusqu'à $0,75 \mathrm{~m}$ ), l'autre superficiel et alimenté par les pluies.

L'évolution du rapport $L E_{m} / R_{n m}$ du sous-bois est très différente. La transpiration de la molinie représente une part beaucoup plus importante du rayonnement net absorbé à son niveau; d'autre part, ce rapport diminue moins en cours de saison que celui du houppier. Ceci est sans doute dû à la part de rayonnement net parvenant jusqu'au sous-bois, qui, quoique non négligeable, est relativement modérée, et qui, de plus, diminue au fur et à mesure de l'été ( $37 \%$ du rayonnement net total au jour 163 , le $R_{n m}$ de la journée équivaut à l'évaporation de $3,2 \mathrm{~mm}$ d'eau, $21 \%$ au jour 269 , équivalant à $1,0 \mathrm{~mm}$ ). La demande climatique est peu élevée et s'atténue donc fortement en cours de saison, ce qui permet à la molinie, malgré le stress hydrique, de rester turgescente et de maintenir une transpiration importante, qui reste toujours de l'ordre de la moitié de l'évapotranspiration totale.

L'effet de la pluie sur la fermeture stomatique de la molinie n'apparaît pas (fig 7). Cependant, il semble (Loustau, données non publiées) qu'il n'en soit pas ainsi pour les mesures faites à l'aide de la "cage à transpiration". D'autres données sont nécessaires pour répondre à cette question, mais, de toute façon, le stress hydrique affecte beaucoup moins l'estimation de l'évapotranspiration de la molinie que celle des pins (figs 4,5 et 6).

La méthode basée sur l'anémométrie sonique est d'un emploi beaucoup plus dé- licat que celle du flux de sève : nous n'avons présenté ici que les journées de mesures offrant des résultats indiscutables. Même si nous pouvons en utiliser quelques autres, elles resteront discontinues. En revanche, le flux de sève, couplé à des mesures de routine de rayonnement, température et pression de vapeur d'eau peut nous permettre de suivre pratiquement sans interruption l'évolution des conductances de surface (à condition d'éliminer les périodes de stockage et de destockage de sève dans les troncs) et du rapport $L E / R_{n}$ sur toute une saison de végétation, donc de répondre à la plupart des interrogations présentées dans cet article. En revanche, pour le sous-bois, l'anémométrie sonique reste malgré tout la moins lourde : heureusement il semble aussi que les phénomènes y soient plus faciles à décrire.

\section{RÉFÉRENCES}

Amadou M (1989) Estimation de l'évaporation d'un couvert de pin maritime (Pinus pinaster Ait) par la méthode du bilan d'énergiefluctuations de température et de vitesse verticale du vent. Rapport de DAA, ENSA de Rennes

André JC, Goutorbe JP, Perrier A (1986) Hapex-Mobilhy : A hydrologic atmospheric experiment for the study of water budget and evaporation flux at the climatic scale. Bull Am Meteorol Soc 67, 138-144

Biltoft CA, Gaynor JE (1987) Comparison of two types of sonic anemometers and fastresponse thermometers. Proc Am Meteorol Soc Meet New Orleans, USA, January 1987

Brejon de la Vergnée G (1988) Optimisation d'un réseau de mesures neutroniques à l'aide de la théorie des variables régionalisées dans une parcelle de pin maritime (Pinus pinaster). Mémoire de DAA, ENSA de Rennes

Campbell GS, Unsworth MH (1979) An inexpensive sonic anemometer for eddy correlation. J Appl Meteorol 18, 1072-1077 
Choisnel E (1985) Un modèle agrométéorologique opérationnel de bilan hydrique utilisant les données climatiques. In: Les besoins en eau des cultures. INRA, Versailles, 115-132

Cochard $H$ (1988) Utilisation d'un système de type enceinte fermée pour l'estimation de la transpiration journalière d'un sous-bois de pin maritime. (Pinus Pinaster Ait) Mémoire de DEA, Univ Paris-Sud, Orsay

Diawara A (1990) Échanges d'énergie et de masse à l'intérieur et au-dessus d'une forêt de pins des Landes. Thèse, université Blaise-Pascal (Clermont II)

Diawara A, Loustau D, Berbigier P (1990) Comparison of two methods for estimating the evaporation of a Pinus pinaster (Ait) stand: sap flow and energy balance with sensible heat flux measurements by an eddy covariance method. Agric For Meteorol (sous presse)

El Hadj Moussa F (1989) Circulation de l'eau dans un écosystème de pin maritime en Landes de Gascogne. Thèse de doctorat, université de Pau et des Pays de l'Adour

Gash JHC, Shuttleworth WJ, Lloyd CR, André JC, Goutorbe JP, Gelpe J (1989) Micrometeorological measurements in Les Landes forest during Hapex-Mobilhy. Agric For Meteorol 46, 131-147

Granier A (1985) Une nouvelle méthode pour la mesure du flux de sève dans le tronc des arbres. Ann Sci For 42, 193-200

Granier A (1987a) Mesure du flux de sève dans le tronc du Douglas par une nouvelle méthode thermique. Ann Sci For 44, 1-14

Granier A (1987b) Evaluation of transpiration in a Douglas-fir stand by means of sap-flow measurements. Tree Physiol 3, 309-320

Granier A, Bobay V, Gash JHC, Gelpe J, Saugier B, Shuttleworth WJ (1990) Vapour flux density and transpiration rate comparisons in a stand of Maritime pine (Pinus pinaster Ait) in Les Landes forest. Agric For Meteorol $51,309-319$

Jarvis PG (1981) Stomatal conductance, gaseous exchanges and transpiration. In: Plants and their atmospheric environment (Grace J, Ford ED, Jarvis PG, eds). Blackwell, Oxford, 175-204

Jarvis PG, Steward JB (1979) Evaporation of water from plantation forest. In: The ecology of even-aged forest plantations (Ford ED, Malcom DC, Atterson J, eds). Inst Terrestrial Ecol, NERC, Cambridge, 327-350

Lohammar T, Larsson S, Linder S, Falk SO (1980) FAST-simulation models of gaseous exchanges in Scots Pine. In: Structure and function of northern coniferous forest. An ecosystem study (Persson T, ed). Ecol Bull 32, 505-523

Loustau D, Granier A, El Hadj Moussa F (1990) Évolution saisonnière du flux de sève dans un peuplement de pins maritimes. Ann Sci For 599-618

Mc Cauchey JH, Saxton WL (1988) Energy balance storage terms in a mixed forest. Agric For Meteorol 44, 1-18

McNaughton KG, Black TA (1973) Evaporation from a forest: a micrometeorological study. Water Resour Res 9, 1579-1590

Monteith JL (1973) Principles of environmental physics. Edward Arnold, London

Schulze ED, Cermak J, Matyssek R, Penka M, Zimmermann R, Vascek F, Gries W, Kucera J (1985) Canopy transpiration and water fluxes in the xylem of the trunk of Larix and Picea trees - a comparison of xylem flow, porometer and cuvette measurements. $\mathrm{Oe}$ cologia (Berlin) 66, 475-483

Shuttleworth WJ (1989) Micrometeorology of temperate and tropical forests. Philos Trans $R$ Soc Lond B Biol Sci B324, 299-334

Shuttleworth WJ, Gash JHC, Lloyd CL, Moore CJ, Wallace JS (1988) An integrated meteorological system for evaporation measurement. Agric For Meteorol 43, 295-317

Stewart JB (1988) Modelling surface conductance of pine forest. Agric For Meteorol 43, 19-35

Waring RH (1976) Environmental control of leaf water conductance in conifers. Can $J$ For Res 6, 104-112

Waring RH, Whitehead D, Jarvis PG (1979) The contribution of stored water to transpiration in Scots Pine. Plant Cell Environ 2, 309-317

Whitehead D, Jarvis PG, Waring RH (1984) Stomatal conductance transpiration, and resistance to water uptake in a Pinus sylvestris spacing experiment. Can J For Res 14 , $692-700$ 